\title{
openheart Erratum: Opportunistic detection of atrial fibrillation using blood pressure monitors: a systematic review
}

Kane SA, Blake JR, McArdle FJ, et al. Opportunistic detection of atrial fibrillation using blood pressure monitors: a systematic review. Open Heart 2016;3:e000362. doi:10.1136/ openhrt-2015-000362corr1

Table 2 Summary of the results from the QUADAS-2 tool for each of the included papers.

\begin{tabular}{|c|c|c|c|c|c|c|c|}
\hline \multirow[b]{2}{*}{ Study } & \multicolumn{4}{|c|}{ RISK OF BIAS } & \multicolumn{3}{|c|}{ APPLICABILITY CONCERNS } \\
\hline & $\begin{array}{l}\text { PATIENT } \\
\text { SELECTION }\end{array}$ & $\begin{array}{l}\text { INDEX } \\
\text { TEST }\end{array}$ & $\begin{array}{l}\text { REFERENCE } \\
\text { STANDARD }\end{array}$ & $\begin{array}{l}\text { FLOW } \\
\text { AND } \\
\text { TIMING }\end{array}$ & $\begin{array}{l}\text { PATIENT } \\
\text { SELECTION }\end{array}$ & $\begin{array}{l}\text { INDEX } \\
\text { TEST }\end{array}$ & $\begin{array}{l}\text { REFERENCE } \\
\text { STANDARD }\end{array}$ \\
\hline Wiesel et al. ${ }^{11}$ & + & - & ? & - & - & - & ? \\
\hline Stergiou et al. ${ }^{12}$ & + & - & ? & ? & - & - & + \\
\hline Wiesel et al. ${ }^{13}$ & + & - & + & + & + & + & + \\
\hline Marazzi et al. ${ }^{14}$ & + & + & + & - & + & - & + \\
\hline Wiesel et al. ${ }^{15}$ & + & + & + & + & - & + & - \\
\hline Kearley et al. ${ }^{16}$ & + & + & + & + & + & + & + \\
\hline Wiesel et al. ${ }^{17}$ & + & + & + & + & + & - & + \\
\hline
\end{tabular}

+, Low Risk; -, High Risk; ?, Unclear Risk.

The incorrect table 2 was published with the paper. Please find the correct table now provided below:

Open Heart 2016;0:e000362corr1. doi:10.1136/openhrt-2015-000362corr1

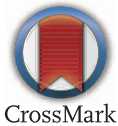

